\title{
Características Morfológicas y Perfil Antropométrico en Mujeres que Practican Pilates Clásico y Mat Clásico
}

\author{
Morphological Characteristics and Anthropometric Profile of Women \\ who Practice Reformer Pilates and Traditional Mat Pilates
}

\author{
Raquel Vaquero-Cristóbal"; Fernando Alacid**; Francisco Esparza-Ros*; \\ José María Muyor**** \& Pedro Ángel López-Miñarro*****
}

VAQUERO-CRISTÓBAL, R.; ALACID, F.; ESPARZA-ROS, F.; MUYOR J. M. \& LÓPEZ-MIÑARRO, P. A. Características morfológicas y perfil antropométrico en mujeres que practican Pilates clásico y mat clásico. Int. J. Morphol., 32(2):695-702, 2014.

RESUMEN: El objetivo de este trabajo fue describir y comparar las características antropométricas, somatotipo y proporcionalidad en mujeres que practicaban mat clásico y Pilates clásico. Setenta y nueve mujeres, 39 que realizaban Pilates clásico (edad: 45,10 $\pm 8,32$

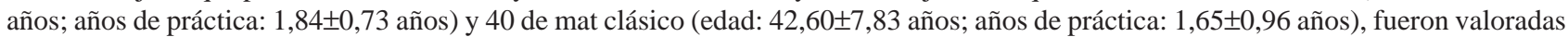
usando una batería de 41 medidas antropométricas. Se calculó el somatotipo, los valores Z utilizando la estrategia Phamton de proporcionalidad y la composición corporal siguiendo la estrategia de cinco componentes de Kerr. Ambos grupos de mujeres obtuvieron valores similares en la mayoría de las variables antropométricas, valores $\mathrm{Z}$ de proporcionalidad, porcentajes de composición corporal y en el somatotipo, clasificado como meso-endomórfico. En conclusión, la práctica de mat clásico o Pilates clásico en mujeres no genera diferencias en las variables antropométricas, la composición corporal y el somatotipo.

PALABRAS CLAVE: Antropometría; Ejercicio físico; Pilates.

\section{INTRODUCCIÓN}

En los últimos años el método Pilates está teniendo una gran acogida a nivel mundial, lo que ha provocado que se hayan publicado numerosos manuales y libros relacionados con esta actividad. No obstante, estos carecen, por lo general, del suficiente rigor científico, existiendo pocos estudios que hayan demostrado que la práctica del método Pilates produce mejoras reales en la condición física saludable (Bernardo, 2007; Rogers \& Gibson, 2009; Sekendiz et al., 2007), en la percepción de la imagen corporal (CruzFerreira et al., 2011), la cual está muy desvirtuada en los países desarrollados como consecuencia de la interiorización de modelos prodelgadez, sobre todo entre las mujeres (Vaquero-Cristóbal et al., 2013), y en la composición corporal de quien lo practica. En esta línea, se ha encontrado que se reduce la grasa corporal total o de los miembros (Baltaci et al., 2005; Çakmakçi, 2011; Cruz-Ferreira et al., 2009; García \& Aznar, 2011; Pan, 2006; Rogers \& Gibson), el sumatorio de pliegues y los pliegues individuales (Çakmakçi), aumenta la masa magra (Çakmakçi; Cruz-Ferreira et al., 2009), disminuye el índice de masa corporal (IMC) (Çakmakçi), el peso (Baltaci et al.; Çakmakçi; Pan), la relación cintura-cadera
(Çakmakçi; García \& Aznar), la circunferencia de la cintura (Çakmakçi; Pan), de la cadera, del brazo y mesoesternal (Pan). Por otro lado, algunas investigaciones encuentran que el método Pilates no provoca grandes cambios en la composición corporal del sujeto (Jago et al., 2006; Segal et al., 2004; Sekendiz et al.). Estas investigaciones presentan ciertas limitaciones, destacando que incluyen una muestra muy pequeña, intervenciones cortas o frecuencias e intensidades bajas. Además, en todos ellos, las intervenciones se llevan a cabo en la práctica de mat clásico, habiendo un único estudio de intervención en Pilates clásico (Pilates trabajando en el reformer) con una población de adultos que realizaban una única sesión semanal de 60 minutos durante 24 semanas (Segal et al.). Esta frecuencia es, según los criterios de la Organización Mundial de la Salud (2012) y los parámetros del estudio de Olson et al. (2004) realizado en practicantes del método Pilates, insuficiente para lograr cambios en la composición corporal, el estado de fitness o la salud. Por tanto, hasta la actualidad, no existen estudios que hayan analizado los efectos de la práctica del Pilates clásico en mujeres adultas sin patologías crónicas y con un volumen de práctica suficiente.

* Cátedra de Traumatología del Deporte, Universidad Católica de San Antonio de Murcia, Murcia, España.

** Facultad de Ciencias de la Actividad Física y el Deporte, Universidad Católica de San Antonio de Murcia, Murcia, España.

*** Área de Expresión Corporal. Facultad de Ciencias de la Educación, Universidad de Almería, Almería, España.

***** Departamento de Expresión Plástica, Musical y Dinámica. Universidad de Murcia, Murcia, España. 
Aunque los fundamentos del mat clásico y del Pilates clásico son similares, hay grandes diferencias entre ambas modalidades. Mientras que en mat clásico se trabaja en el suelo con implementos, en el Pilates clásico se trabaja con una maquina (el reformer) sobre la que se desliza una plataforma que se mueve a lo largo de ella mediante railes, proporcionando una guía en la ejecución técnica al practicante (Latey, 2001).

Diversas investigaciones refieren diferencias en las características antropométricas en función de la posición de juego en deportes colectivos como el fútbol (Hencken \& White, 2006), voleibol (Toledo et al., 2010), baloncesto (Goncetas \& Landor, 2005) y en función de la disciplina practicada en deportes individuales como el atletismo (Vucetic et al., 2008), la natación (Carter \& Ackland, 1994) el remo (Kerr et al., 2007) y el piragüismo (Alacid et al., 2012). La práctica de un tipo u otro de Pilates también podría generar diferencias en las características antropométricas de quien realiza esta actividad. Puesto que hasta la actualidad no existen estudios sobre la composición corporal en practicantes de Pilates clásico, los objetivos de la presente investigación fueron: 1) describir las características antropométricas, somatotipo y proporcionalidad de mujeres practicantes del método Pilates; 2) comparar los resultados en función de si practican mat clásico o Pilates clásico.

\section{MATERIAL Y MÉTODO}

Un total de 79 mujeres adultas, con edades comprendidas entre 30 y 60 años, practicantes del método Pilates, participaron en este estudio. Las características de la muestra se presentan en la Tabla I. El criterio de inclusión fue tener una experiencia en la práctica de Pilates de 1 a 4 años y realizar un volumen de entrenamiento de dos horas semanales, repartidas en dos días a la semana. Las clases eran impartidas por personal cualificado para la enseñanza del método Pilates, una gran experiencia formativa (título de instructor de Pilates y Diplomatura en Fisioterapia) y profesional (al menos un año de experiencia cualificado Pilates).

El estudio fue aprobado por la Comisión de Bioética Institucional. Previamente a las mediciones, todas las participantes fueron informadas sobre los procedimientos y firmaron, voluntariamente, un consentimiento informado.
Las variables fueron tomadas por un antropometrista acreditado de nivel II, siguiendo las indicaciones descritas por la ISAK (International Society for the Advancement of Kinanthropometry) (Stewart et al., 2011). Las medidas se tomaron dos o tres veces, si la diferencia entre las dos primeras era superior al $5 \%$ en pliegues y al $1 \%$ en el resto de medidas, tomando la media o la mediana, respectivamente, para realizar los análisis posteriores.

El instrumental fue calibrado con antelación para evitar errores en la medición. La temperatura del laboratorio donde se realizaron las mediciones fue estandarizada a $24^{\circ} \mathrm{C}$.

Para la determinación del peso se utilizó una báscula SECA 862 (SECA, Alemania) de 100 g de precisión; para los pliegues un plicómetro Harpenden (British Indicators, UK) de 0,2 mm de precisión; para la envergadura y los perímetros una cinta métrica inextensible milimetrada Lufkin W606PM (Lufkin, EE.UU.); y para la talla, talla sentado, alturas, longitudes y diámetros, un antropómetro SiberHegner GPM (GPM, Suiza), un paquímetro Holtain (Holtain Ltd., Reino Unido) y un segmómetro Cescorf (Ltda., Brasil), todos ellos con una precisión de $0,1 \mathrm{~cm}$.

Las ecuaciones de Carter \& Heath (1990) se utilizaron para calcular cada uno de los componentes del somatotipo y Estrategia del Phantom (Ross \& Marfell-Jones, 1991) para la determinación de los valores $\mathrm{Z}$ de cada una de las variables. También se calculó el índice de masa corporal, los sumatorios de seis y ocho pliegues cutáneos y los perímetros corregidos. Para determinar la composición corporal se utilizó la estrategia de cinco componentes de Kerr (1988).

Los resultados fueron analizados en primera instancia de forma conjunta $y$, posteriormente, de manera independiente para las practicantes de mat clásico y Pilates clásico. Para el análisis de datos se realizó un análisis descriptivo de cada una de las variables. Para comparar los resultados en función de la modalidad de Pilates practicada se determinó previamente si la muestra seguía una distribución normal utilizando el test de Shapiro-Wilk. Si las variables mostraban una distribución normal, se aplicó una prueba t para variables independientes, mientras que para aquéllas cuya distribución no seguía una distribución normal se aplicó la prueba U de Mann-Whitney. Para todos los test estadísticos el nivel de significación se situó en un valor de $\mathrm{p}<$

Tabla I. Características de la población.

\begin{tabular}{lcccc}
\hline & Mat + Pilates & Mat clásico & Pilates clásico & $\begin{array}{c}\text { Valor } \boldsymbol{p} \text { al comparar los } \\
\text { grupos de mat y Pilates }\end{array}$ \\
\hline Número & 79 & 40 & 39 & \\
Edad & $43,84 \pm 8,12$ & $42,60 \pm 7,83$ & $45,10 \pm 8,32$ & 0,650 \\
Años de práctica & $1,74 \pm 0,85$ & $1,65 \pm 0,96$ & $1,84 \pm 0,73$ & 0,223 \\
\hline
\end{tabular}


0,05. Todas las pruebas fueron realizadas usando el paquete estadístico SPSS para Windows (versión 15.0).

\section{RESULTADOS Y DISCUSIÓN}

La Tabla II presenta las características antropo-métricas y las diferencias entre las mujeres que practicaban Pilates clásico y mat clásico. Se encontraron diferencias significativas en el diámetro trasverso del tórax, siendo mayores los valores de las practicantes de mat clásico, mientras que en el perímetro de la muñeca y de la pierna corregida, así como en la longitud de la mano las practicantes de Pilates clásico obtuvieron valores significativamente mayores.

Por el contrario, no se encontraron diferencias significativas entre ambos grupos en los valores de peso y altura. $\mathrm{Al}$ depender el IMC directamente de ambas variables tampoco se encontraron diferencias significativas en este parámetro, situándose en normopeso para ambos grupos (Tabla III). El IMC del presente estudio, al igual que el sumatorio de seis pliegues, fue ligeramente superior al mostrado en otro estudio con practicantes del método Pilates (García \& Aznar). Esto se podría relacionar con diferencias en las muestras, pues el estudio de García \& Aznar incluía en su muestra un grupo de hombres y mujeres, sin separar entre géneros al analizar los datos.

Siguiendo con las comparaciones entre los valores de las variables antropométricas del presente estudio y los encontrados por Çakmakçi se encuentra que las mujeres del presente estudio mostraron valores inferiores en el peso, IMC, circunferencia de la cintura, ratio cintura/cadera, así como en los pliegues del bíceps, tríceps, subescapular y cresta iliaca tanto antes como después de un programa de intervención de 8 semanas, el cual consistió en la realización de una hora de mat clásico, cuatro días a la semana. Estas diferencias podrían deberse a que Çakmakçi seleccionó una población de mujeres obesas y principiantes. Se ha demostrado que con la práctica del método Pilates se trabajan los músculos recto abdominal, trasverso y oblicuos del abdomen (Critchley et al., 2010; Rogers \& Gibson; Sekendiz et $a l$.), lo cual puede incidir de manera directa en la circunferencia de la cintura y la proporcion cintura/cadera y podría justificar que las mujeres del presente estudio tengan valores inferiores en este parámetro, pues llevaban al menos un año de práctica en esta modalidad deportiva.

El perfil de seis y ocho pliegues se muestra en las Figuras 1 y 2 respectivamente. No se han observado diferencias significativas entre grupos en ninguno de los pliegues.

Los valores Z de proporcionalidad Phanthom de am- bos grupos se muestran en la Tabla IV. Se hallaron valores similares en ambos grupos. Únicamente se encontró que las mujeres que practicaban Pilates clásico presentaban valores significativamente superiores en la envergadura, la longitud de la mano y el perímetro de la pierna corregido. El hecho de que no hayan grandes diferencias entre los grupos puede deberse a que todas estas medidas de proporcionalidad son obtenidas tomando como referencia las proporciones de un modelo de referencia (Phantom) y la talla del sujeto a estudiar. Al ser la talla de ambos grupos muy similar, se encuentran en las puntuaciones del valor $\mathrm{Z}$ prácticamente las mismas diferencias significativas que se hallaban al comparar los valores de las medidas en ambos grupos.

El somatotipo medio de ambos grupos (Fig. 3) se correspondió con el morfotipo meso-endomorfo, siendo los valores similares entre las practicantes del mat clásico y Pilates clásico (Tabla III). La dispersión morfogénica media del somatotipo (somatotype attitudinal mean), como una medida de la dispersión media de los somatotipos individuales respecto al somatotipo medio del grupo (Pilates clásico o mat clásico), fue de 1,21 $\pm 0,58$ en las practicantes de mat clásico y de 1,64 $\pm 1,00$ en Pilates clásico. En base a estos resultados se encontró que las practicantes de Pilates clásico presentaron una mayor heterogeneidad que las de mat clásico (Fig. 3).

$\mathrm{Al}$ analizar la composición corporal mediante la estrategia de cinco componentes de Kerr se observó que no hay diferencias significativas entre ambos grupos en cualquiera de los componentes estimados (masa de la piel, masa grasa, masa ósea, masa muscular y masa residual), encontrándose valores superiores en el porcentaje de masa grasa y el de masa muscular que en la masa de la piel, la masa ósea y la masa residual (Tabla III). Por otro lado, aunque en este modelo penta-compartimental no se utiliza el peso total en el cálculo de ninguna de las masas, el error medio respecto a la suma de las masas individuales derivadas fue de $3,36 \pm 1,80 \mathrm{~kg}$, aspecto que refuerza el uso de esta metodología para la determinación de la composición corporal.

El porcentaje de grasa de las participantes de este estudio fue superior al encontrado en otros trabajos de investigación (García \& Aznar; Segal et al., 2004), lo cual puede deberse a que en uno de ellos la muestra estaba compuesta por grupos de hombres y mujeres, sin diferenciar entre géneros (García \& Aznar, 2010) y en el otro las participantes eran mucho más jóvenes que las del presente estudio (Segal et al.). Además, se utilizaron diferentes métodos para estimar el porcentaje de grasa corporal, decantándose por la bio-impedancia en el estudio de Segal et al. y por la fórmula de Faulkner a partir de 4 pliegues corporales en el de García \& Aznar. Por el contrario, el porcentaje de grasa corporal 
Tabla II. Comparación y descripción de las características antropométricas de las mujeres practicantes del método Pilates.

\begin{tabular}{|c|c|c|c|c|}
\hline Variable & $\begin{array}{c}\text { Mat + Pilates } \\
(n=79)\end{array}$ & $\begin{array}{c}\text { Mat clásico } \\
(n=40)\end{array}$ & $\begin{array}{c}\text { Pilates clásico } \\
\quad(n=39)\end{array}$ & $\mathbf{p}-$ valor $*$ \\
\hline Peso $(\mathrm{kg})$ & $63,59 \pm 7,74$ & $64,10 \pm 8,63$ & $63,08 \pm 6,78$ & 0,637 \\
\hline Sumatorio de 6 pliegues $(\mathrm{mm})$ & $105,94 \pm 22,11$ & $107,60 \pm 19,79$ & $104,23 \pm 24,42$ & 0,504 \\
\hline Sumatorio de 8 pliegues $(\mathrm{mm})$ & $131,53 \pm 28,85$ & $134,20 \pm 26,69$ & $128,79 \pm 31,02$ & 0,410 \\
\hline Talla $(\mathrm{cm})$ & $164,62 \pm 4,47$ & $164,90 \pm 4,18$ & $164,33 \pm 4,79$ & 0,798 \\
\hline Talla sentado $(\mathrm{cm})$ & $84,66 \pm 6,92$ & $84,20 \pm 3,38$ & $85,13 \pm 9,29$ & 0,295 \\
\hline Envergadura (cm) & $165,65 \pm 5,92$ & $165,50 \pm 5,85$ & $165,79 \pm 6,06$ & 0,798 \\
\hline PL Tríceps (mm) & $16,14 \pm 4,90$ & $15,30 \pm 4,81$ & $17,00 \pm 4,89$ & 0,156 \\
\hline PL Subescapular (mm) & $14,34 \pm 4,12$ & $14,50 \pm 3,90$ & $14,18 \pm 4,37$ & 0,608 \\
\hline PL Bíceps (mm) & $8,66 \pm 3,21$ & $8,90 \pm 2,49$ & $8,41 \pm 3,83$ & 0,351 \\
\hline PL Cresta iliaca (mm) & $17,03 \pm 5,11$ & $17,80 \pm 5,72$ & $16,23 \pm 4,33$ & 0,262 \\
\hline PL Supraespinal (mm) & $14,52 \pm 4,25$ & $14,90 \pm 3,80$ & $14,13 \pm 4,69$ & 0,354 \\
\hline PL Abdominal (mm) & $20,05 \pm 4,59$ & $20,50 \pm 4,55$ & $19,59 \pm 4,63$ & 0,397 \\
\hline PL Muslo (mm) & $23,42 \pm 6,75$ & $24,10 \pm 7,40$ & $22,72 \pm 6,02$ & 0,738 \\
\hline PL Pierna medial (mm) & $17,42 \pm 5,97$ & $18,20 \pm 6,10$ & $16,62 \pm 5,79$ & 0,844 \\
\hline D Biacromial $(\mathrm{cm})$ & $34,43 \pm 1,93$ & $34,60 \pm 1,76$ & $34,26 \pm 2,11$ & 0,525 \\
\hline D Biileocrestal $(\mathrm{cm})$ & $25,85 \pm 2,19$ & $26,20 \pm 2,21$ & $25,49 \pm 2,15$ & 0,483 \\
\hline D Transverso del tórax (cm) & $23,80 \pm 2,10$ & $24,30 \pm 2,07$ & $22,28 \pm 2,02$ & $\mathbf{0 , 0 2 2}$ \\
\hline D Anteroposterior del tórax (cm) & $19,39 \pm 1,83$ & $19,50 \pm 1,51$ & $19,28 \pm 2,12$ & 0,157 \\
\hline D Biepicondíleo del húmero $(\mathrm{cm})$ & $6,06 \pm 0,29$ & $6,00 \pm 0,10$ & $6,13 \pm 0,40$ & 0,056 \\
\hline D Bicondíleo del fémur $(\mathrm{cm})$ & $8,99 \pm 0,74$ & $8,80 \pm 0,60$ & $9,18 \pm 0,82$ & 0,888 \\
\hline D Biestiloideo $(\mathrm{cm})$ & $4,97 \pm 0,62$ & $4,80 \pm 0,40$ & $5,15 \pm 0,74$ & 0,642 \\
\hline PR Cabeza $(\mathrm{cm})$ & $54,68 \pm 1,47$ & $54,70 \pm 1,43$ & $54,67 \pm 1,52$ & 0,817 \\
\hline PR Cuello (cm) & $31,22 \pm 1,93$ & $31,00 \pm 1,28$ & $31,44 \pm 2,43$ & 0,888 \\
\hline PR Brazo relajado (cm) & $27,20 \pm 2,41$ & $27,10 \pm 2,65$ & $27,31 \pm 2,16$ & 0,578 \\
\hline PR Brazo corregido (cm) & $22,10 \pm 2,52$ & $22,30 \pm 2,79$ & $21,90 \pm 2,23$ & 0,343 \\
\hline PR Brazo contraído $(\mathrm{cm})$ & $28,29 \pm 2,33$ & $28,10 \pm 2,41$ & $28,49 \pm 2,27$ & 0,712 \\
\hline PR Antebrazo $(\mathrm{cm})$ & $23,53 \pm 1,70$ & $23,20 \pm 1,34$ & $23,87 \pm 1,97$ & 0,164 \\
\hline PR Muñeca $(\mathrm{cm})$ & $14,47 \pm 0,86$ & $14,10 \pm 0,54$ & $14,85 \pm 0,96$ & 0,006 \\
\hline PR Mesoesternal (cm) & $89,95 \pm 5,94$ & $90,40 \pm 6,87$ & $89,49 \pm 4,85$ & 0,844 \\
\hline PR Cintura $(\mathrm{cm})$ & $73,44 \pm 6,55$ & $74,00 \pm 7,21$ & $72,87 \pm 5,84$ & 0,694 \\
\hline PR Cadera $(\mathrm{cm})$ & $97,46 \pm 6,04$ & $97,90 \pm 7,155$ & $97,00 \pm 4,70$ & 0,651 \\
\hline $\begin{array}{l}\text { PR Muslo }(1 \mathrm{~cm} \text { por debajo de la } \\
\text { línea glútea })(\mathrm{cm})\end{array}$ & $54,19 \pm 4,57$ & $54,40 \pm 5,20$ & $53,97 \pm 3,86$ & 0,636 \\
\hline PR Muslo medio (cm) & $48,71 \pm 3,35$ & $48,80 \pm 3,53$ & $48,62 \pm 3,20$ & 0,622 \\
\hline PR Muslo corregido $(\mathrm{cm})$ & $41,33 \pm 2,83$ & $41,10 \pm 2,80$ & $41,56 \pm 2,88$ & 0,737 \\
\hline PR Pierna (máx.) (cm) & $34,53 \pm 3,06$ & $34,00 \pm 3,53$ & $35,08 \pm 2,42$ & 0,160 \\
\hline PR Pierna corregido $(\mathrm{cm})$ & $29,13 \pm 3,46$ & $28,40 \pm 4,08$ & $29,87 \pm 2,52$ & 0,019 \\
\hline PR Tobillo $(\mathrm{cm})$ & $20,87 \pm 1,20$ & $20,70 \pm 1,11$ & $21,05 \pm 1,27$ & 0,825 \\
\hline L Brazo $(\mathrm{cm})$ & $31,33 \pm 1,63$ & $31,60 \pm 1,93$ & $31,05 \pm 1,21$ & 0,247 \\
\hline L Antebrazo (cm) & $24,01 \pm 1,11$ & $24,00 \pm 1,10$ & $24,03 \pm 1,13$ & 0,990 \\
\hline L Mano $(\mathrm{cm})$ & $18,99 \pm 0,88$ & $18,70 \pm 0,64$ & $19,28 \pm 0,99$ & 0,008 \\
\hline H Ileoespinal (cm) & $92,86 \pm 3,83$ & $92,90 \pm 4,18$ & $92,82 \pm 3,50$ & 0,392 \\
\hline H Troncantérea $(\mathrm{cm})$ & $80,86 \pm 13,64$ & $80,20 \pm 15,59$ & $81,54 \pm 11,48$ & 0,206 \\
\hline L Muslo $(\mathrm{cm})$ & $40,06 \pm 5,35$ & $38,90 \pm 2,58$ & $41,26 \pm 7,01$ & 0,142 \\
\hline H Tibialelaterale $(\mathrm{cm})$ & $44,04 \pm 2,07$ & $44,30 \pm 2,22$ & $43,77 \pm 1,91$ & 0,735 \\
\hline L Pierna (medial) (cm) & $34,19 \pm 2,38$ & $33,90 \pm 2,76$ & $33,97 \pm 1,94$ & 0,663 \\
\hline L Pie $(\mathrm{cm})$ & $22,81 \pm 2,02$ & $23,20 \pm 1,80$ & $22,41 \pm 2,18$ & 0,080 \\
\hline
\end{tabular}

fue menor que el hallado por Çakmakçi en un programa de intervención con mujeres obesas. De cualquier forma, todas estas comparaciones deben interpretarse con cautela debido a la gran variedad de fórmulas, pliegues seleccionados y 


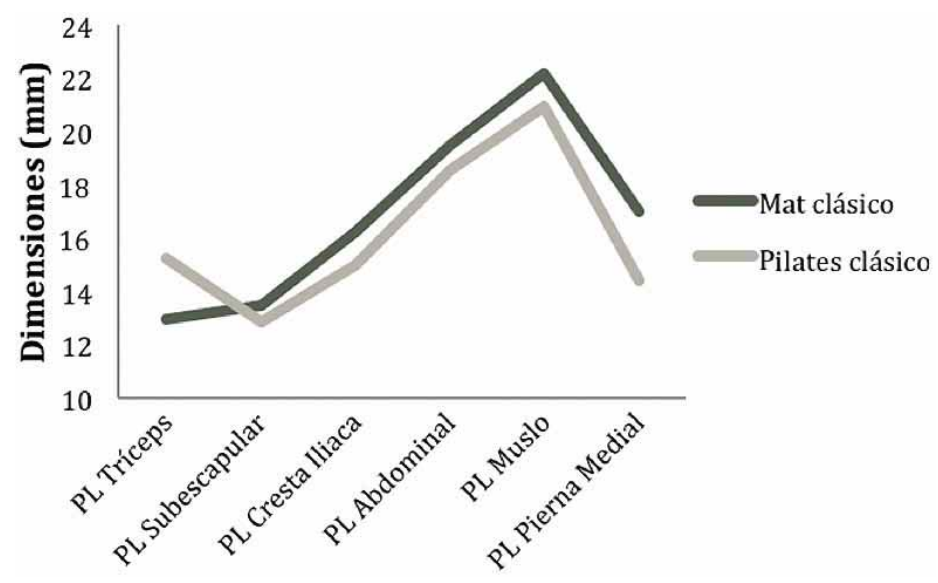

Fig. 1. Perfil de seis pliegues. PL= Pliegue.

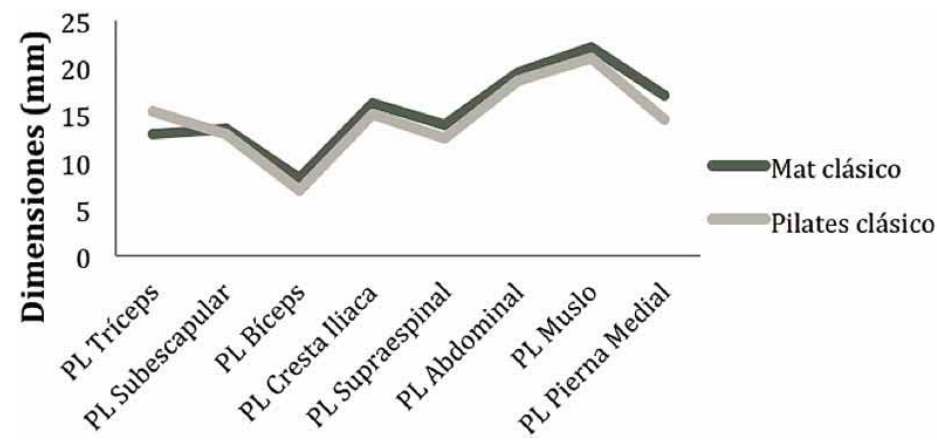

Fig. 2. Perfil de ocho pliegues. PL= Pliegue.

Tabla III. Comparación y descripción de la proporcionalidad (Valores Z) de las practicantes de Pilates.

\begin{tabular}{lrrrc}
\hline Variable & Mat + Pilates & Mat clásico & Pilates clásico & p - valor * \\
\hline IMC & $23,41 \pm 2,74$ & $23,50 \pm 3,04$ & $23,31 \pm 2,44$ & 0,782 \\
Ratio & $0,75 \pm 0,02$ & $0,75 \pm 0,02$ & $0,75 \pm 0,03$ & 0,898 \\
Endomorfia & $4,57 \pm 1,08$ & $4,45 \pm 0,74$ & $4,69 \pm 1,34$ & 0,233 \\
Mesomorfia & $3,65 \pm 1,11$ & $3,20 \pm 0,82$ & $4,10 \pm 1,18$ & 0,158 \\
Ectomorfia & $1,87 \pm 0,91$ & $1,90 \pm 0,87$ & $1,85 \pm 0,96$ & 0,223 \\
Masa de la piel & $5,66 \pm 0,37$ & $5,66 \pm 0,41$ & $5,66 \pm 3,32$ & 0,296 \\
Masa ósea (\%) & $9,31 \pm 1,58$ & $9,36 \pm 1,54$ & $9,27 \pm 1,64$ & 0,556 \\
Masa grasa (\%) & $34,90 \pm 4,23$ & $35,40 \pm 4,00$ & $34,38 \pm 4,45$ & 0,666 \\
Masa muscular & $35,33 \pm 3,41$ & $34,60 \pm 3,54$ & $36,08 \pm 3,14$ & 0,336 \\
Masa residual (\%) & $9,49 \pm 2,02$ & $9,90 \pm 0,95$ & $9,08 \pm 2,66$ & 0,098 \\
Masa de la piel & $3,59 \pm 0,49$ & $3,60 \pm 0,49$ & $3,59 \pm 0,49$ & 0,855 \\
Masa ósea (kg) & $5,90 \pm 1,06$ & $6,00 \pm 1,01$ & $5,79 \pm 1,12$ & 0,806 \\
Masa grasa (kg) & $22,13 \pm 3,59$ & $22,50 \pm 3,48$ & $21,74 \pm 3,71$ & 0,296 \\
Masa muscular & $22,67 \pm 4,10$ & $22,40 \pm 4,66$ & $22,95 \pm 3,47$ & 0,430 \\
Masa residual & $6,06 \pm 1,68$ & $6,30 \pm 1,28$ & $5,82 \pm 1,99$ & 0,768 \\
Diferencia & $3,36 \pm 11,80$ & $4,59 \pm 12,48$ & $2,09 \pm 11,08$ & \\
respecto al peso & & & & \\
\hline
\end{tabular}

Datos expresados como Media \pm Desviación Típica. $\mathrm{PL}=$ Pliegue, $\mathrm{PR}=$ perímetro, $\mathrm{D}=$ diámetro, $\mathrm{L}=$ Longitud, H= Altura. *=valor de la comparación entre mat clásico y Pilates clásico. 
Tabla III. Comparación y descripción del somatotipo, composición corporal, índice de masa corporal y ratio cintura/cadera entre las practicantes de mat clásico y Pilates clásico.

\begin{tabular}{|c|c|c|c|c|}
\hline Variable & $\begin{array}{c}\text { Mat + Pilates } \\
(n=79)\end{array}$ & $\begin{array}{c}\text { Mat clásico } \\
(n=40)\end{array}$ & $\begin{array}{c}\text { Pilates clásico } \\
\quad(n=39)\end{array}$ & $\mathbf{p}-$ valor $*$ \\
\hline Z Peso & $0,77 \pm 1,01$ & $0,80 \pm 1,09$ & $0,74 \pm 0,93$ & 0,525 \\
\hline$Z$ Talla sentado & $-0,54 \pm 1,43$ & $-0,70 \pm 0,91$ & $-0,38 \pm 1,81$ & 0,583 \\
\hline$Z$ Envergadura & $-0,18 \pm 0,59$ & $-0,30 \pm 0,64$ & $-0,05 \pm 0,51$ & 0,047 \\
\hline Z PL Tríceps & $0,29 \pm 1,15$ & $0,10 \pm 1,05$ & $0,49 \pm 1,23$ & 0,095 \\
\hline Z PL Subescapular & $-0,44 \pm 0,90$ & $-0,40 \pm 0,92$ & $-0,49 \pm 0,88$ & 0,664 \\
\hline Z PL Bíceps & $0,39 \pm 1,62$ & $0,40 \pm 1,29$ & $0,38 \pm 1,92$ & 0,341 \\
\hline Z PL Cresta iliaca & $-0,81 \pm 0,80$ & $-0,80 \pm 0,88$ & $-0,82 \pm 0,72$ & 0,279 \\
\hline Z PL Supraespinal & $-0,16 \pm 0,99$ & $-0,10 \pm 0,95$ & $-0,23 \pm 1,03$ & 0,475 \\
\hline Z PL Abdominal & $-0,62 \pm 0,64$ & $-0,60 \pm 0,67$ & $-0,64 \pm 0,62$ & 0,402 \\
\hline Z PL Muslo & $-0,30 \pm 0,86$ & $-0,20 \pm 0,99$ & $-0,41 \pm 0,71$ & 0,091 \\
\hline Z PL Pierna medial & $0,41 \pm 1,23$ & $0,50 \pm 1,21$ & $0,31 \pm 1,26$ & 0,296 \\
\hline Z D Biac romial & $-1,37 \pm 0,98$ & $-1,40 \pm 0,92$ & $-1,33 \pm 1,06$ & 0,291 \\
\hline Z D Biileocrestal & $-1,20 \pm 1,26$ & $-1,00 \pm 1,19$ & $-1,41 \pm 1,31$ & 0,131 \\
\hline Z D Trasnverso del tórax & $-2,00 \pm 1,29$ & $-1,70 \pm 1,36$ & $-2,31 \pm 1,15$ & 0,108 \\
\hline Z D Anteroposterior del tórax & $1,97 \pm 1,42$ & $2,01 \pm 1,15$ & $1,85 \pm 1,66$ & 0,086 \\
\hline Z D Biepicondíleo del húmero & $-0,34 \pm 0,83$ & $-0,50 \pm 0,67$ & $-0,18 \pm 0,94$ & 0,133 \\
\hline Z D Bicondíleo del fémur & $0,51 \pm 1,46$ & $-0,70 \pm 0,91$ & $-0,31 \pm 1,86$ & 0,305 \\
\hline Z D Biestiloideo & $0,11 \pm 1,95$ & $-0,40 \pm 1,12$ & $0,64 \pm 2,44$ & 0,361 \\
\hline Z PR Cabeza & $0,51 \pm 1,65$ & $0,50 \pm 1,71$ & $0,51 \pm 1,62$ & 0,840 \\
\hline Z PR Cuello & $-1,46 \pm 1,19$ & $-1,60 \pm 0,92$ & $-1,31 \pm 1,41$ & 0,167 \\
\hline$Z$ PR Brazo relajado & $0,58 \pm 1,12$ & $0,60 \pm 1,21$ & $0,56 \pm 1,04$ & 0,409 \\
\hline Z PR Brazo corregido & $2,27 \pm 1,20$ & $2,30 \pm 1,28$ & $2,23 \pm 1,13$ & 0,606 \\
\hline Z PR Brazo contraído & $-0,05 \pm 1,02$ & $-0,10 \pm 0,95$ & $0,00 \pm 1,10$ & 0,293 \\
\hline Z PR Antebrazo & $-0,48 \pm 1,11$ & $-0,70 \pm 0,79$ & $-0,26 \pm 1,35$ & 0,196 \\
\hline Z PR Muñeca & $-1,85 \pm 1,11$ & $-2,40 \pm 0,81$ & $-1,28 \pm 1,09$ & 0,157 \\
\hline Z PR Mesoesternal & $1,13 \pm 1,28$ & $1,30 \pm 1,36$ & $0,95 \pm 1,19$ & 0,106 \\
\hline Z PR Cintura & $0,92 \pm 1,51$ & $1,00 \pm 1,56$ & $0,85 \pm 1,47$ & 0,794 \\
\hline$Z$ PR Cadera & $1,11 \pm 1,19$ & $1,20 \pm 1,34$ & $1,03 \pm 1,03$ & 0,788 \\
\hline Z PR Muslo ( $1 \mathrm{~cm}$ por debajo de la línea glútea) & $0,09 \pm 1,23$ & $0,20 \pm 1,41$ & $-0,03 \pm 1,01$ & 0,606 \\
\hline Z PR Muslo medio & $-0,59 \pm 0,80$ & $-0,60 \pm 0,81$ & $-0,59 \pm 0,81$ & 0,847 \\
\hline Z PR Pierna (máx.) & $0,19 \pm 1,36$ & $-0,10 \pm 1,59$ & $0,49 \pm 1,02$ & 0,156 \\
\hline Z PR Pierna corregido & $1,86 \pm 1,70$ & $1,50 \pm 2,03$ & $2,23 \pm 1,18$ & $\mathbf{0 , 0 3 3}$ \\
\hline Z PR Tobillo & $-0,15 \pm 0,81$ & $-0,40 \pm 0,67$ & $0,10 \pm 0,88$ & 0,595 \\
\hline Z L Brazo & $-0,06 \pm 0,89$ & $0,10 \pm 1,05$ & $-0,23 \pm 0,66$ & 0,856 \\
\hline Z L Antebrazo & $0,09 \pm 0,72$ & $0,00 \pm 0,64$ & $0,18 \pm 0,79$ & 0,136 \\
\hline Z L Mano & $0,96 \pm 0,91$ & $0,60 \pm 0,49$ & $1,33 \pm 1,08$ & $\mathbf{0 , 0 0 0}$ \\
\hline Z H Ileoespinal & $0,34 \pm 0,59$ & $0,30 \pm 0,64$ & $0,38 \pm 0,54$ & 0,274 \\
\hline Z H Troncantérea & $-0,72 \pm 3,10$ & $-0,90 \pm 3,46$ & $-0,54 \pm 2,71$ & 0,232 \\
\hline Z L Muslo & $0,15 \pm 2,16$ & $-0,30 \pm 0,91$ & $0,62 \pm 2,88$ & 0,177 \\
\hline Z H Tibialelaterale & $0,05 \pm 0,61$ & $0,10 \pm 0,70$ & $0,00 \pm 0,51$ & 0,440 \\
\hline Z L Pierna (medial) & $-0,61 \pm 0,99$ & $-0,50 \pm 1,13$ & $-0,72 \pm 0,82$ & 0,090 \\
\hline Z L Pie & $-1,71 \pm 1,68$ & $-1,40 \pm 1,58$ & $-2,03 \pm 1,73$ & 0,364 \\
\hline
\end{tabular}




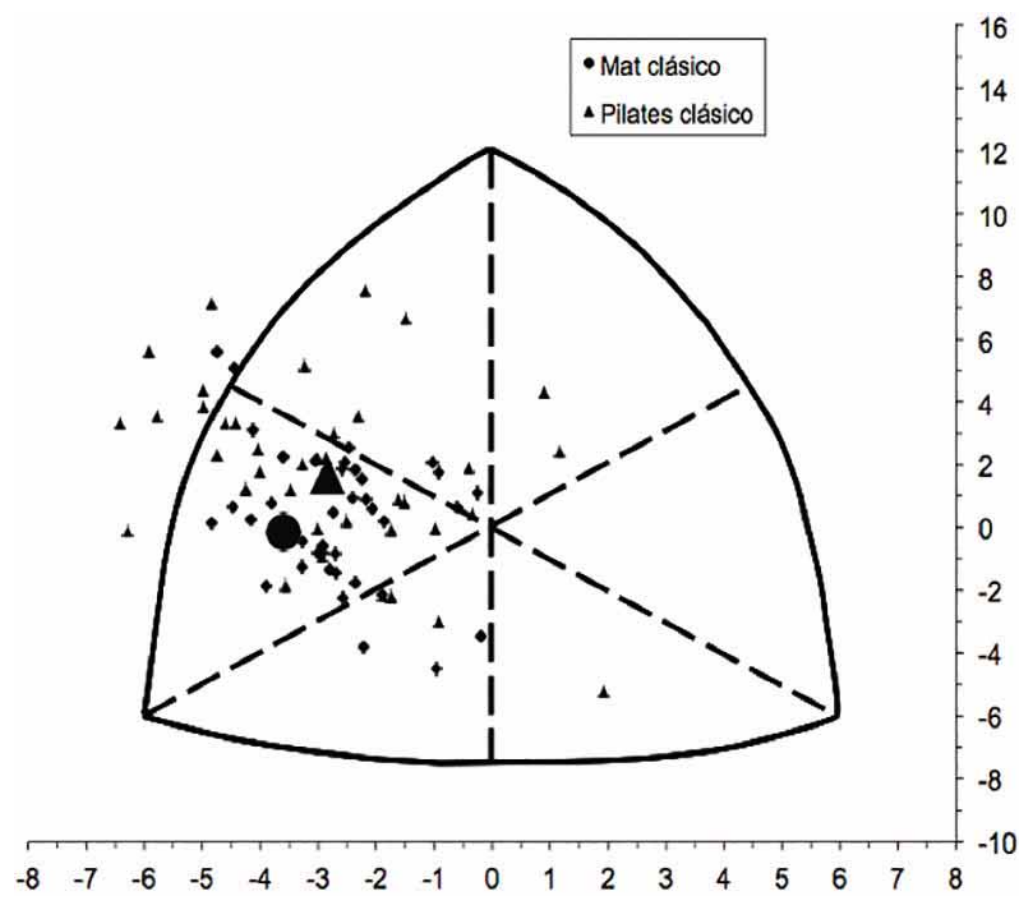

Fig. 3. Somatocarta de las mujeres practicantes de mat clásico y Pilates clásico en función de los valores del somatotipo.

técnicas utilizadas en la obtención de los mismos para el cálculo posterior del porcentaje de grasa.

En conclusión, ambos grupos mostraron unos valores similares en todas las variables antropométricas, de proporcionalidad y de composición corporal, siendo los porcentajes de grasa y muscular muy similares, mientras que el IMC se situó en normopeso. Tanto las practicantes de mat clásico como las de Pilates clásico presentaron un somatotipo medio similar, clasificado como meso-endomórfico. Este trabajo aporta información normativa sobre las características antropométricas de las mujeres practicantes de Pilates y mat clásico que pueden utilizarse para describir la morfología de esta población, ya que se han llevado a cabo pocos trabajos de investigación sobre esta práctica física y en ninguno de ellos se han comparado a personas que practicaran diferentes especialidades de Pilates.

\section{AGRADECIMIENTOS}

Los autores agradecen su colaboración a todos los centros que participaron en este estudio, especialmente al centro Movement Pilates y Salud, así como a las mujeres que participaron en el estudio.

VAQUERO-CRISTÓBAL, R.; ALACID, F.; ESPARZAdescribed as meso-endomorph in both groups. In conclusion, both groups showed similar values in all anthropometric dimensions, proportional values, body composition and somatotype.

KEY WORDS: Anthropometry; Physical exercise; Pilates.

SUMMARY: The aim of this study was to describe and compare the kinanthropometric characteristics, somatotype and proportionality between women who perform reformer Pilates and traditional mat Pilates. Seventy-nine women, 39 who practice reformer Pilates (age: $45.10 \pm 8.32$ years; years of practice: $1.84 \pm 0.73$ years) and 40 who do mat Pilates (age: $42.60 \pm 7.83$ years; years of practice: $1.65 \pm 0.96$ years), were assessed using a battery of 41 anthropometric dimensions. Somatotypes, Phantom Z-scores and body composition with the strategy of five components of Kerr were calculated. Both groups showed similar values of anthropometric dimensions, phantom Zscores, values of body composition and mean somatotypes. Somatotype was best

\section{REFERENCIAS BIBLIOGRÁFICAS}

Alacid, F.; Muyor, J. M.; Vaquero, R. \& López-Miñarro, P. A. Características morfológicas y maduración en mujeres kayakistas jóvenes de aguas tranquilas y slalom. Int. J. Morphol., 30(3):895-901, 2012.

Baltaci, G.; Bayrakci Tunay, V.; Yakut, E. \& Vardar, N. A comparison of two different exercises on the weight loss in the treatment of knee osteoarthritis: Pilates exercises versus clinical-based physical therapy. Osteoarthritis Cartilage, 13(Suppl. A):S141, 2005.

Bernardo, L. M. The effectiveness of Pilates training in healthy adults: an appraisal of the research literature. J. Bodyw. Mov. Ther., 11(2):106-10, 2007.

Çakmakçi, O. The effect of 8 week pilates exercise on body composition in obese women. Coll. Antropol., 35(4):1045-50, 2011.

Carter, J. E. L. \& Ackland, T. R. Kinanthropometry in aquatic 
sports. A study of world class athletes. Champaign, Human Kinetics, 1994.

Carter, J. E. L. \& Heath, B. H. Somatotyping: development and application. Cambridge, Cambridge University Press, 1990.

Critchley, D. J.; Pierson, Z. \& Battersby, G. Effect of pilates mat exercises and conventional exercise programmes on transversus abdominis and obliquus internus abdominis activity: pilot randomised trial. Man. Ther., 16(2):183-9, 2011.

Cruz-Ferreira, A. I. C.; Pereira, C. L. N. \& Fernandes, J. A. Effects of three months of Pilates-based exercise in women on body composition. Med. Sci. Sports. Exerc., 41(5):16-7, 2009.

Cruz-Ferreira, A.; Fernandes, J.; Gomes, D.; Bernardo, L. M.; Kirkcaldy, B. D.; Barbosa, T. M. \& Silva, A. Effects of Pilatesbased exercise on life satisfaction, physical self-concept and health status in adult women. Women Health, 51(3):240-55, 2011.

García, P. T. \& Aznar, L. S. Práctica del método Pilates: cambios en composición corporal y flexibilidad en adultos sanos. Apunts Med. Esport., 46(169):17-22, 2011.

Goncetas, A. \& Landor, A. Morphological and physiological parameters in relation to playing position of high level basketball players. Paper Anthropol., 14:42-52, 2005.

Hencken, C. \& White, C. Anthropometric assessment of Premiership soccer players in relation to playing position. Eur. J. Sport Sci., 6(4):205-11, 2006.

Jago, R.; Jonker, M. L.; Missaghian, M. \& Baranowski, T. Effect of 4 weeks of Pilates on the body composition of young girls. Prev. Med. 42(3):177-80, 2006.

Kerr, D. A. An anthropometric method for fractionation of skin, adipose, bone, muscle and residual tissue masses, in males and females age 6 to 77 years. MSc Kinesiology Thesis. British Columbia, Simon Fraser University, 1988.

Kerr, D. A.; Ross, W. D.; Norton, K.; Hume, P.; Kagawa, M. \& Ackland, T. R. Olympic lightweight and open-class rowers possess distinctive physical and proportionality characteristics. J. Sports Sci., 25(1):43-53, 2007.

Latey, P. The Pilates method: history and philosophy. J. Bodyw. Mov. Ther., 5(4):275-82, 2001.

Olson, M. S.; Williford, H. N.; Martin, R. St.; Ellis, M.; Woolen, E. \& Esco, M. R. The energy cost of a basic, intermediate, and advanced Pilates' mat workout. Med. Sci. Sports Exerc., 36(5):S357, 2004.

Organización Mundial de la Salud. La actividad física en los adultos mayores. En: Estrategia mundial sobre régimen alimentario, actividad física y salud. 2012. Disponible en: http:// www.who.int/dietphysicalactivity/factsheet_olderadults/es/ index.html

Pan, F. Effects of Pilate's exercise program on physical and mental health of community-dwelling middle to older adults with obesity. MSc Thesis, Kaohsiung, Fooyin University, 2006.

Rogers, K. \& Gibson, A. L. Eight-week traditional mat Pilates training-program effects on adult fitness characteristics. Res. Q. Exerc. Sport., 80(3):569-74, 2009.

Ross, W. D. \& Marfell-Jones, M. J. Kinanthropometry. En: MacDougall, J. D.; Wenger, H. A. \& Green, H. J. (Eds.). Physiological testing of the high performance athlete. $2^{\text {nd }}$ ed. Champaign, Human Kinetics, 1991. pp.223-308.

Sekendiz, B.; Altun, O.; Korkusuz, F. \& Akin, S. Effects of Pilates exercise on trunk strength, endurance and flexibility in sedentary adult females. J. Bodyw. Mov. Ther., 11(4):318-26, 2007.

Segal, N. A.; Hein, J. \& Basford, J. R. The effects of Pilates training on flexibility and body composition: an observational study. Arch. Phys. Med. Rehabil., 85(12):1977-81, 2004.

Stewart, A.; Marfell-Jones, M.; Olds, T. \& de Ridder, H. International standards for anthropometric assessment. LowerHutt, ISAK, 2011.

Toledo, F. C. L.; Roquetti, F. P. \& Fernandes, F. J. Análisis del perfil antropométrico de jugadores de la selección brasileña de voleibol infanto juvenil. Int. J. Morphol., 28(4):1035-41, 2010 .

Vaquero-Cristóbal, R.; Alacid, F.; Muyor, J. M. \& López-Miñarro, P. A. Imagen corporal; revisión bibliográfica. Nutr. Hosp., 28(1):27-35, 2013.

Vucetic, V.; Matkovic, B. R. \& Sentija, D. Morphological differences of elite Croatian track-and-field athletes. Coll. Antropol., 32(3):863-8, 2008.

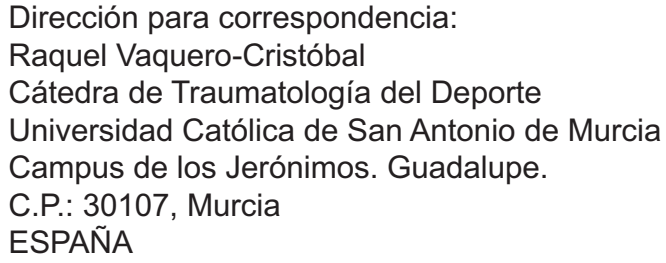

Email: rvaquero@ucam.edu

Recibido : 13-02-2013

Aceptado: 10-01-2014 